\title{
Variations
}

Variations Revue internationale de théorie critique

12 | 2008

Wärmestrom

\section{Masse et fascisme}

\section{Micha Brumlik}

Traducteur : Alexander Neumann

\section{OpenEdition}

\section{Journals}

Édition électronique

URL : http://journals.openedition.org/variations/242

DOI : 10.4000/variations.242

ISSN : 1968-3960

Éditeur

Les amis de Variations

Édition imprimée

Date de publication : 21 décembre 2008

Référence électronique

Micha Brumlik, « Masse et fascisme », Variations [En ligne], 12 | 2008, mis en ligne le 01 janvier 2012, consulté le 02 mai 2019. URL : http://journals.openedition.org/variations/242 ; DOI : 10.4000/

variations. 242

Ce document a été généré automatiquement le 2 mai 2019.

Les ami•e•s de Variations 


\title{
Masse et fascisme
}

\author{
Micha Brumlik
}

Traduction : Alexander Neumann

\section{NOTE DE L'ÉDITEUR}

Traduit de l'allemand

Première publication sur www.theoriecritique.com, «Wärmestrom, le courant chaud en sciences humaines ", Hiver 2008/2009, pp. 58-65

L'article correspond à un chapitre du livre de Micha Brumlik, Sigmund Freud - Der Denker des 20. Jahrhunderts, Beltz Verlag, 2006, pp.195. Nous remercions l'auteur et l'éditeur de leur aimable autorisation à publier une traduction française.

1 L'époque du fascisme s'est annoncée comme une époque des masses, plus précisément des masses fascinées ou séduites par des chefs (Führer). Lorsque l'ouvrage majeur du sociologue Max Weber fut publié en 1921 à titre posthume, l'un de ses chapitres fut déjà consacré à la «domination charismatique » pour nommer, à travers le concept de " charisme », les qualités d'une personnalité « qui font qu'elle est perçue comme un chef, qui le font apparaitre comme un émissaire de Dieu ou comme une personne exemplaire, donc dotée de forces et de qualités (le talent) surnaturelles ou surhumaines, ou du moins inhabituelles. $»^{1}$

2 La domination charismatique a toujours de bonnes chances de s'exercer lorsque les formes habituelles du pouvoir politique sont débordées par des phénomènes de crise sociale qui font qu'il ne fonctionne plus comme avant ou qu'il est mis hors service. À la différence de la domination bureaucratique qui s'assoit sur la légitimité de règles explicites, et de la domination traditionnelle dont la légitimité est liée à l'existence de coutumes incontestées, la domination charismatique ne tire sa légitimité que de «l'épreuve », donc de la « reconnaissance délibérée du chef par les dominés, qui naît de leur dévotion, leur admiration du héros, leur confiance envers lui $»{ }^{2}$ 
Weber n'avait pas besoin de faire l'expérience, ni des mouvements de masse modernes, ni des chefs fascistes (les Führer) pour être en mesure de décliner cette forme de domination sur un plan théorique, même si son analyse de la domination qu'exerça Bismarck, publiée en 1918, pointe déjà dans cette direction. Le sociologue était conscient du fait que la forme de charisme qu'il analysait, ce "pouvoir révolutionnaire ", était inséré dans une époque encore marquée par la tradition, et il pensait que ce charisme pouvait organiser une transformation, donc « un changement des directions fondamentales des actions et des idées, au cours d'une réorientation complète de toutes les attitudes envers les différents domaines de la vie et du monde en général ».3

Le concept de «Führer » a été diffusé à partir des années 1890 à travers le mouvement de jeunesse nationaliste d'Allemagne, entouré d'une vision du monde où se mêlaient des théories philosophiques, sociales et esthétiques, flanquées de l'idée que la «ligue » (Bund) serait une forme de vie supérieure à celle de la société moderne existante. Dans la réalité sociale de l'après-guerre, ce concept de Führer entrait alors en correspondance avec celui de masse qu'il devait diriger, phénomène que le psychologue français Gustave Le Bon (1841-1931) avait décrit de façon saisissante et sobre dès 1895. L'étude au sujet de «L'insurrection des masses » qui fut publiée en 1929 par le philosophe espagnol Ortega y Gasset (1883-1955), ainsi que l'œuvre du sociologue nord-américain David Riesman sur «La foule solitaire », témoignent du fait que la «masse » était alors perçue comme un phénomène aussi nouveau qu'inquiétant, du moins aux yeux des observateurs sociologiques issues de la noblesse et de la bourgeoisie. La masse n'attirait non seulement l'attention grâce à son ampleur quantitative tout à fait inhabituelle, mais encore par le fait qu'elle faisait apparaître des hommes qui échappaient à l'habitus partiellement autonome des bourgeois ou des nobles, en sortant dans le même temps de la léthargie qui caractérisait les cerfs, les servants et le quatrième état de la société féodale, traditionnelle. Il apparaissait que les masses étaient capables d'articuler leurs intérêts, si ce n'était pas à travers leur propre bouche, du moins en se fiant à celle des chefs qu'elles acclamaient.

5 En 1920, Sigmund Freud passait des vacances à Bad Gastein dans les Alpes, où il a déclaré avoir réuni des matériaux pour une étude sur la psychologie des masses, sans pourtant être prêt à investir «ces problèmes profonds ». Son biographe Peter Gay décrit Freud comme quelqu'un qui pratiquait un « libéralisme politique désuet », qui considérait avec un certain mépris ce "peuple débile " (dixit Freud) et qui ne s'intéressait pas autre mesure à la politique, en préférant appliquer sa psychanalyse aux dirigeants et aux masses. ${ }^{4}$ Cette interprétation suggère que Freud aurait raisonné sur la base des catégories fonctionnelles que son biographe estime essentielles; mais il est tout aussi légitime de penser que Freud s'est intéressé à la chose politique à partir d'un autre angle, qui lui donnait accès aux conditions subconscientes de l'action politique et qui lui permettait de les percer à jour. Ainsi, l'ouvrage «Le malaise dans la civilisation » de 1930 se présente comme un traité qui ne craint pas la comparaison aux classiques de la philosophie politique, d'autant plus qu'il les mobilise et qu'il en prolonge les arguments.

6 En 1920, il s'agissait d'abord d'établir une "psychologie de masse», en saisissant les individus dans leur qualité de membres "d'un peuple, d'une caste, d'un état, d'une institution ou d'une foule humaine, et qui s'organise sous la forme d'une masse à un moment donné $» .^{5}$

7 Après la première Guerre Mondiale, les masses et leurs leaders ont visiblement occupé le tout premier plan des opinions publiques en Europe; qu'il s'agisse des agitateurs de 
l'après-guerre immédiat en Allemagne et en Autriche ou qu'il s'agisse des chefs communistes de la Russie révolutionnaire, ou encore sous la forme spectaculaire du politicien italien Benito Mussolini, passé du socialisme à une attitude belliciste, nationaliste et anti-syndicale, faisant fureur à chacune de ses prestations publiques, malgré des performances électorales médiocres. Les «ligues» de combat de Mussolini, lancées en mars1919, ont submergé la classe ouvrière avec une vague de violence saignante en 1920, à travers leurs actions contre les syndicats et les occupations d'usine. ${ }^{6}$

Victor Klemperer, à qui l'on doit le journal aussi impressionnant qu'oppressant d'un juif allemand harcelé sous le nazisme, a assisté en 1932 à la projection d'un film à l'occasion du dixième anniversaire de la marche sur Rome, à Dresde, film dans lequel l'écrivain a vu et entendu « il Duce ». Son observation se distingue par le souci du détail : « On voit bien, comment le Duce se gonfle littéralement à bloc, à chaque nouvelle phrase, pour ensuite se relâcher, en répétant ainsi une expression mimique et gestuelle d'une énergie et d'une tension extrême ; on entend le vibrato qui évoque un prêche passionné, rituel, rythmé par des phrases courtes qu'il balance successivement, tel des fragments d'une liturgie, et qui font réagir tout un chacun émotionnellement sans lui demander le moindre effort mental, même et surtout s'il n'en saisit pas le sens. $»^{7}$

«Et surtout s'il n'en saisit pas le sens...»; l'observation expérimentale de Klemperer confirme ainsi l'analyse du rapport entre masse et Führer que Freud a développé, à partir de sa réception critique des écrits de Gustave Le Bon. Selon Freud, l'homme pris dans la masse se distingue par une activité affective exacerbée, une performance intellectuelle restreinte, ce qui peut donc aboutir à une nette désinhibition. Freud constate aussi que la plupart des travaux concernant la psychologie de masse dont il a pris connaissance mobilisent le concept de "pouvoir évocateur» (Suggestion), mais en acceptant ce phénomène comme un fait incompressible. Trente ans après ses propres expériences en la matière, lorsque Freud avait été jeune médecin à la Salpêtrière parisienne, où il avait assisté aux exercices d'évocation du célèbre Bernheim, il revient donc sur cette thématique, avec la ferme intention de venir à bout de ce phénomène dont il entend analyser les fondements. En activant sa théorie libidinale ici, il compte examiner le fort lien affectif qui unit les hommes au sein de la masse, afin de comprendre quel besoin pousse les humains à se conformer aux Autres plutôt que de s'y opposer. Deux exemples particulièrement parlants qui font figure de « masse artificielle » doivent servir à illustrer la question: L'Eglise et l'Armée. L'un des enjeux étant d'être en mesure de distinguer les masses disposant d'un chef et les masses sans chef. L'organisation de ces deux masses ne repose non seulement sur des postes et des hiérarchies, mais encore sur le mirage qui veut «qu'une autorité suprême existe - Jésus-Christ pour l'Eglise catholique, le Commandeur pour l'Armée - qui aime chaque individu composant la masse de façon égale. $»^{8}$

10 L'église catholique et l'Armée servent ainsi de modèle exemplaire à Freud (mais on peut mettre en doute la pertinence de son analyse lorsqu'il définit l'autorité suprême comme étant le Christ et non pas le Pape, ce qui peut être vrai d'un point de vue théologique, mais non pas sur le plan organisationnel de l'église catholique avec ses hiérarchies bien établies dont Freud ne tient pas compte).

11 L'église d'un côté, l'Armée de l'autre, auraient ceci de différent que l'église serait démocratique dans sa structure affective, puisque chaque membre est égal devant le Christ en bénéficiant de son amour de la même façon. Par conséquent, «il est 
incontestable que le lien de chacun envers Jésus établit également le lien unissant les membres entre eux. ${ }^{9}$

L'Armée suit une logique similaire quoique non identique : Ici le lien prend la forme de la camaraderie qui s'établit à travers l'amour commun envers une figure paternelle, à la différence décisive près que la structuration hiérarchique de l'Armée fait apparaître des figures paternelles à tous les niveaux. Freud s'oppose au passage à un argument issu de l'histoire des idées, selon lequel les Armées tireraient leur unité de l'amour envers des idées, tels que la patrie ou l'honneur. Il conteste la supposition qui voudrait que des idées puissent remplacer une relation respectueuse des chefs militaires envers leurs subordonnés, en rappelant les graves névroses provoquées par la première Guerre Mondiale qui ont été constatées au sein de l'Armée allemande.

Le mécanisme psychique crucial qui conduit les membres d'une masse à adhérer à un chef / Führer et à se lier entre eux, repose sur l'une des deux relations possibles à travers lesquelles des humains peuvent se mettre en lien avec d'autres: Ou bien sous la forme d'un objet de leur désir (Objektwahl), ou bien sous la forme d'un Autre à qui l'on voudrait ressembler. Ces deux choix possibles sont exposés à l'aide de la première enfance et de sa constellation oedipale, quand le garçon veut posséder la mère, tout en voulant ressembler au père. Freud conclut que la forme originaire de la formation des sentiments n'est pas la "Objektwahl» (fixation sur un objet), mais "l'identification ». ${ }^{10}$ Il s'agit d'une forme affective qui peut remplacer une fixation libidinale sur un objet qui aurait échoué, lorsque l'individu intègre son objet du désir dans sa propre personnalité (le Moi), ce qui conduit ensuite à un attachement affectif de la personne à d'autres qui présentent une même orientation, sans qu'il perçoive ces personnes directement comme des objets de son propre désir sexuel (un procédé bien connu en psychopathologie). L'identification permet aussi bien l'empathie que la construction interne d'un idéal personnel (idéal du Moi). Le souhait de vouloir ressembler à un Autre peut naturellement conduire au dérapage, notamment lorsque la personne ne se contente plus d'intégrer l'objet de son admiration en un idéal du Moi interne, mais commence à le désirer en tant que tel. Cela apparait parfois lorsque des personnes tombent amoureux, surtout chez les adolescents. Tout se joue finalement dans la question de savoir si «l'objet vient se poser à l'endroit du Moi ou s'il vient remplacer l'idéal du Moi $»^{11}$, dit autrement si la personne aimée est considérée comme quelqu'un à qui l'individu veut ressembler à tout prix, ou si les souhaits et volontés de la personne aimée vont complètement remplacer les motivations propres de l'individu concerné.

Cette situation n'est pas très éloignée de l'hypnose, proche du sentiment amoureux en ce qu'elle se caractérise par «la dévotion soumise, la docilité et l'absence de critique ${ }^{12}$ L'identification et le sentiment amoureux ont ceci en commun qu'ils représentent tous deux des pulsions sexuelles entravées, qui peuvent se manifester lorsque la satisfaction immédiate est rendue impossible. Freud résout l'énigme du pouvoir évocateur en saisissant la corrélation structurelle de l'hypnose et de la formation des masses, qui réside dans le fait que les individus sortent de l'entrelacs de complexe de relations complexes qu'ils entretiennent au moment même où la masse prend corps.

L'analogie complète entre l'hypnose et la masse bute cependant sur le fait que l'hypnose contient un aspect complémentaire que le sentiment amoureux ne possède pas (ni l'amour des membres de la masse envers leur chef), à savoir « la paralysie qui intervient dans le rapport d'un surpuissant envers quelqu'un d'impuissant, sans défense $»^{13}$, et qui peut être comparée à l'hypnose en état de choc qu'on trouve chez les animaux. Une masse 
se forme donc à chaque fois qu'un certain nombre d'individus reconnaissent un seul et même objet qu'ils ont mis à la place de leur idéal du Moi, ce qui les incite à s'identifier mutuellement. Cela s'est passé de la sorte dans le nazisme aussi bien que dans le fascisme italien.

En 1922 déjà, le futur «représentant du Führer (Adolf Hitler, Ndt)», Rodolphe Hess, a livré la prise de position suivante dans un article primé au sujet de la question « Quelles qualités doivent être celles de l'Homme qui conduira l'Allemagne à nouveau vers sa gloire passée ? » : "Nous constatons avec fierté qu'il existe un seul être qui se place au-dessus de toute critique, c'est le Führer. Cela vient du fait que chacun sait et sent qu'il a raison et qu'il aura toujours raison. Notre national-socialisme prend racine dans notre loyauté et dévotion absolues envers le Führer (...) Nous sommes convaincus que le Führer répond à une vocation supérieure qui le charge du destin de l'Allemagne. $»^{14}$

La déclaration du nazi Hess témoigne clairement de sa volonté de remplacer sa propre personnalité (le Moi) par celle d'un Autre, de se vouer complètement à un autre Moi que le sien et de renoncer ainsi au contrôle de sa propre vie. Il ne s'agit pas tellement -du moins dans cette citation- d'un document attestant de la séduction de Hess, mais d'un dernier acte volontaire, celui de ne plus vouloir assumer le fait d'être un être autonome. À l'inverse, cela montre que la séduction ne peut pas aboutir si elle ne rencontre pas la volonté d'être séduit - tout comme dans le domaine de l'amour et de la sexualité.

Freud n'a pas poursuivi la problématique de la formation de la masse moyennant la substitution hypnotique du Moi (et non pas de l'idéal du Moi) par l'objet, mais il a néanmoins poursuivi sa théorie des masses, grâce à l'examen du concept de «l'instinct grégaire » que l'on trouve souvent dans la littérature populaire. Contrairement aux auteurs qui ont utilisé le terme avant lui, il pense que ce phénomène s'explique par la dynamique du développement psychologique, donc il recherche les motivations de ce comportement dans le mode psychique par lequel les petits enfants tentent de maîtriser la jalousie que leur provoque l'arrivée de nouveaux frères ou sœurs. Face à l'impossibilité de monopoliser l'attention des parents, ils se voient obligés de s'identifier les nouveaux enfants et de former un sentiment d'appartenance à une communauté, qui se renforce ensuite grâce au peer group à l'école.

19 Ici prend racine l'aspiration à la justice - qui sera l'un des thèmes du « Malaise dans la civilisation» - et qui comporte un énorme enjeu politique. Freud décrit ainsi l'identification mutuelle des enfants : « Leur première revendication est celle de la justice, du traitement égal de tous (...) S'il est vrai qu'on ne peut pas être soi-même le préféré,

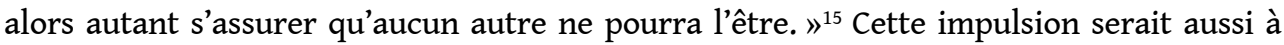
l'origine de «l'esprit communautaire » au sein de la société, qui à ses débuts s'appuierait sur l'envie. Freud reconstruit ensuite le comportement politique qui correspond à cette approche : «Personne ne doit se distinguer, tout le monde doit être pareil et posséder la même chose. La justice sociale signifie que l'on renonce soi-même à bien des choses, pour que les autres soient également obligés d'y renoncer, ou alors pour qu'ils ne puissent pas le réclamer, ce qui revient au même. » Telle est la conviction de Freud en 1920, une année faite de révolutions et de basculements dans toute l'Europe. Il est persuadé que pareille « revendication de la justice sociale constitue la racine de l'éthique sociale et de l'esprit de responsabilité $» .^{16}$

20 En conséquence, les sentiments sociaux résultent de la transformation d'émotions hostiles en une identification plutôt positive aux autres, animée par le souhait de ne pas être plus mal situé qu'eux, à une exception près : Celle du chef, personne supérieure par 
laquelle tous veulent être dominés. Cette compréhension permet de dépasser la construction bancale de "l'instinct grégaire », en reformulant la question à l'aide d'un jeu de mots : Désormais, l'homme pris dans la masse n'apparait plus comme un animal du troupeau (Herdentier), mais comme le membre d'une horde (Hordentier) qui est un groupe d'individus dirigés par un chef. ${ }^{17}$ Ainsi, il arrive à renouer avec ses travaux précédents, réunis dans "Totem et tabou ", tout en préparant le terrain aux thèses qu'il développera plus tard dans "L'homme Moïse et la religion monothéiste », au sujet du rapport entre Moïse et le peuple d'Israël.

Il n'est pas très clair si Freud a totalement rejeté les mécanismes qu'il a lui-même mis à jour. Sans aucun doute, le rationaliste et démystificateur qu'il était ne pouvait que refuser un état social qui se caractérise par la surexcitation affective, l'inhibition de la pensée, la perte de soi et la servitude volontaire. Mais il est permis de poser la question s'il ne percevait pas des aspects positifs dans pareille situation sociale, car « L'homme Moïse » le suggère. (...)

L'ébauche freudienne d'une psychologie de masse peut également être interprétée comme une critique de la culture patriarcale qui exclut systématiquement les femmes et leurs projets de vie, à travers son organisation de masse qui apparait par exemple dans l'église ou l'Armée, en mobilisant des liens affectifs s'apparentant à de l'amour homosexuel entravé. ${ }^{18}$

Finalement, Freud perçoit l'ensemble de ces phénomènes de l'hypnose et de la formation des masses comme étant les rudiments de l'évolution humaine, ces « restes d'un héritage issu de la phylogenèse de la libido humaine $»{ }^{19}$

Cela nous confère une tâche qui résulte de l'expérience de la guerre mondiale et des fascismes: fonder et déployer une culture, une civilisation qui serait en mesure de s'opposer à la régression vers notre propre héritage archaïque, qui nous guette toujours.

\section{NOTES}

1. Max Weber, Wirtschaft und Gesellschaft, Tübingen, 1985, p.140.

2. Weber, «Parlament und Regierung im neugeordneten Deutschland» in : Gesammelte Politische Schriften, Tübingen, 1988, p.311.

3. Weber, op. cit., p.142.

4. Peter Gay, Freud - Eine Biographie für unsere Zeit, Frankfurt/M, 1995, p. 454.

5. S. Freud, Massenpsychologie und Ich-Analyse in : Gesammelte Werke, Vol. XIII, p. 74.

6. S. Reichardt, Faschistische Kampfbünde, Köln, 2003.

7. V. Klemperer, LTI. Notizbuch eines Philologen, Leipzig, 1947, p. 37.

8. Freud, Massenpsychologie, p.102.

9. Freud, op. cit.

10. Freud, op. cit., p.117.

11. Freud, op. cit., p.126.

12. Freud, op. cit.

13. Freud, op. cit., p.127. 
14. C. Schmölders, Hitlers Gesicht. Eine physiognomische Studie, München, 2000, p.104.

15. Freud, op. cit., p.133.

16. Freud, op. cit., p.134.

17. Freud, op. cit., p.135.

18. Freud, op. cit., p. 159.

19. Freud, op. cit., p. 160.

\section{AUTEURS}

\section{MICHA BRUMLIK}

Directeur de l'institut de pédagogie et de socio-psychologie, Francfort 